\title{
Near-field Sensors with Machine Learning for Breast Tumor Detection
}

Maged A. Aldhaeebi

Thamer S. Almoneef

Omar M. Ramahi
University of Waterloo, ON, Canada

Prince Sattam bin Abdulaziz University, Alkharj, Saudi Arabia

University of Waterloo, ON, Canada

\section{Abstract}

In this work, we propose the use of an electrically small novel antenna as a probe combined with a classification algorithm for near field microwave breast tumor detection. The resonant probe is highly sensitive to the changes in the electromagnetic properties of the breast tissues such that the presence of the tumor is estimated by determining the changes in the magnitude and phase response of the reflection coefficient of the sensor. The Principle Component Analysis (PCA) feature extraction method is applied to emphasize the difference in the probe responses for both the healthy and the tumourous cases. We show that when a numerical realistic breast with and without tumor cells is placed in the near field of the probe, the probe is capable of distinguishing between healthy and tumorous tissue. In addition, the probe is able to identify tumors with various sizes placed in single locations.

\section{Introduction}

Breast cancer is considered to be one of the major causes of mortality in women worldwide. The American Cancer Society reported that more than 40,000 woman died in 2018 due to breast cancer[1]. The same society reported that in 2018 , detecting breast tumors at an early stage causes the mortality rates to drop by $39 \%$ of females from 1989 to 2015 [1].

Currently, there are three most common clinical detection techniques which use for breast cancer detection including: X-ray mammography, ultrasound scanning and magnetic resonance imaging (MRI). The current modalities have some limitations for breast cancer detection including ionizing radiation, uncomfortable, low sensitivity and Very expensive [2]. Currently, there are three common clinical imaging and detection modalities used for breast cancer detection: X-ray mammography, magnetic resonance imaging (MRI), and ultrasound scanning $[1,2]$. The current modalities have some limitations including ionizing radiation,Uncomfortable,low sensitivity and Very expensive [2].

To avoid all the limitations in the current breast cancer detection techniques, researchers have shifted their attention to an alternative methodology based on microwaves imaging (MI) which has some advantages for breast cancer detection including inexpensive and non-ionizing modality [2]. MI is based on the contrast in dielectric properties between normal and tumor breast tissues within the microwave band $[3,4]$. Moreover, the significant differentiation in the dielectric properties of normal and tumor breast tissues can be used as the underlying principle for cancer detection using electromagnetic waves $[3,4]$. Lazebnik et al. presented the variations in the microwaves dielectric properties of tumors and normal breast tissues where the value of the permittivity and conductivity of the tumor tissues are higher than normal (healthy) tissues [3]. Recently, Martellosio et al reported the contrast in the dielectric properties of normal and tumors breast tissues which show sharp variation in their dielectric properties in a frequency band from 0.5 to $50 \mathrm{GHz}$ [5].

In this work, we propose an electrically-small probe with an ultra-narrow frequency response. The shift in the magnitude and phase of the reflection coefficient of the probe caused by the presence of a tumor existing inside a human breast is used as the primary detection technique. The main idea of the proposed tumor detection modality introduced here is stemming from two main medical facts. The first is the material composition of the two breasts of a woman ( left and right) are symmetric [6, 7]. The second is the unlikelihood that a woman would develop breast tumors in both breasts at the same time and the unlikelihood that a woman would develop identical breast tumors in both breasts at the same time [7]. The detection modality introduced here calls for the detection test to employ two identical probes positioned symmetrically with respect the two breasts. The responses of the two probes were then recorded (phase and magnitude of the reflection coefficients) and processed using the PCA method to decide whether or not a tumor is present. If the response of the probes from both breasts are identical, then the woman breasts are most likely free of tumors; otherwise, there is a likelihood of the presence of a tumor that can be either benign or cancerous.

\section{Results and Discussion}

The probe consists of a four squares loops with matching network placed at the middle of the probe as shown in Fig. 1. The main reason of using the matching network with the two inductors is to miniaturize the probe so that it resonates at low frequencies. Such low operating frequency is needed to ensure a reasonable penetration level into the breast tissues. The loops were hosted on top of an RO4003C Rogers material with a dielectric constant of of $\varepsilon_{r}=3.38$ and a thickness of $1.54 \mathrm{~mm}$. The side of the probe has a length of $L=50 \mathrm{~mm}$ and width of $w=50 \mathrm{~mm}$. The gaps: $g_{1}=0.5 \mathrm{~mm}$ and $g_{2}=1 \mathrm{~mm}$ play a major role in the sensitivity part of the sensor. The inductors: $L_{1}=0.03 u H$ and $L_{2}=0.24 u H$ play a major role in matching the probe with a $50 \Omega$ feed at the desired resonance frequency of $550 \mathrm{MHz}$ as shown in Fig. 2 which lies within the medical band. The sensor is then simulated in CST Microwave Studio [8].

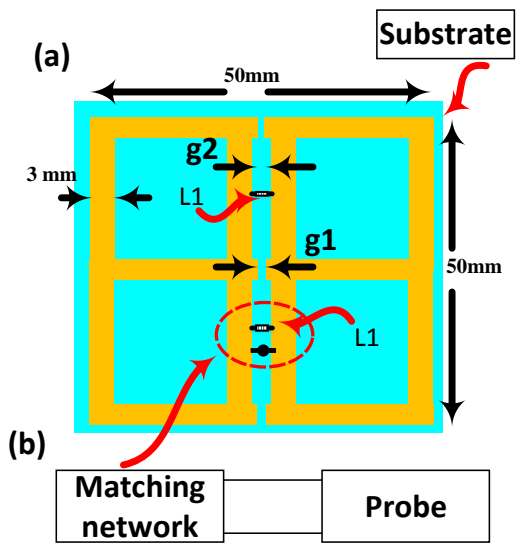

Fig. 1: The proposed electrically small probe consisting of four loops with an extending gap with lumped elements matching network.

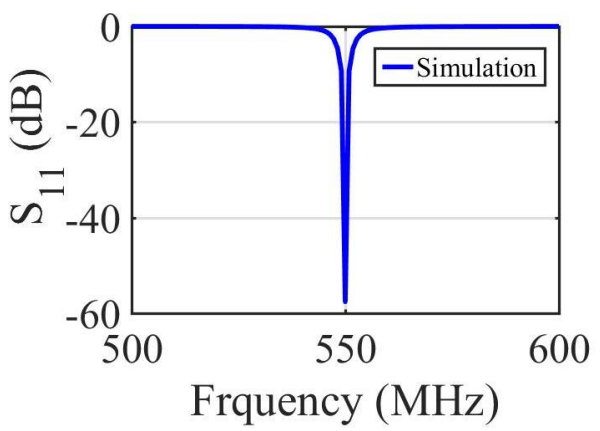

Fig. 2: The reflection coefficient of the probe obtain through simulation.

The proposed sensor was then simulated with a realistic female breast phantom to test its ability to detect the presence of breast tumor cells. In this work, we used the anonymous breast MRI datasets which were obtained from the University of Wisconsin online repository to build the breast phantom in the computer simulation technology $(\mathrm{CST})[9,10]$. The realistic phantom is an 
anatomically realistic (3D) numerical model with dielectric properties derived from T1-weighted MRI images using a piecewise-linear mapping between MRI voxel intensity and the dielectric properties of the breast $[10,11]$. The detests of the numerical phantoms were processed in MALTAB to ingrate the realistic human female breast phantom in the CST. The American College of Radiology (ACR) define four categories of breast composition according to the radiographic density to the density of breast fibrous and glandular tissues including: (I) almost entirely fat, (II) scattered fibroglandular, (III) heterogeneously dense, and (IV) Very dense [10].

Without loss of generality, the class breast phantom used in our work, is the Heterogeneously Dense Breast ID: 062204âĂI ACR classification: Class 3 . The model has $0.5 \times 0.5 \times 0.5$ resolution with $219 \times 243 \times 273$ voxels. After processing the available data in Matlab, the data which includes the breast volume and the single Cole-Cole model for the frequency-dispersive tissues properties were used to construct a numerical model in CST as [10]:

$$
\varepsilon(\omega)=\varepsilon^{\prime}(\omega)-j \varepsilon^{\prime \prime}(\omega)=\varepsilon_{\infty}+\frac{\Delta \varepsilon}{1+(j \omega \tau)^{1-\alpha}}+\frac{\sigma_{s}}{j \omega \varepsilon_{o}}
$$

where $\omega$ is the angular frequency, $\varepsilon^{\prime}(\omega)$ is the frequency-dependant dielectric constant, $\varepsilon^{\prime \prime}(\omega)$ is the frequency-dependant dielectric losses and $\varepsilon_{o}$ is the free space permittivity. The $\varepsilon_{\infty}, \sigma_{s}, \tau$ and $\alpha$ are the Cole-Cole model parameters obtained from the clinical experimental data. The breast model has the physical shape of a real human female breast. Moreover, with a high resolution of $0.5 \mathrm{~mm}$, the model also includes the following eight tissue types: skin, muscle, glandular-1,2,3, and fat-1,2,3 [11]

The probe was calibrated with the normal numerical realistic breast phantom where the proposed probe is placed at a distance of $5 \mathrm{~mm}$ away from the healthy breast phantom as shown in Fig. 3(a). The magnitude and phase of the reflection coefficient of the probe were then recorded at frequency range 630 to $660 \mathrm{MHz}$. Next, a $9 \mathrm{~mm}$ tumor was placed at three different locations inside the healthy breast as shown in Fig. 3(b). The tumor's dielectric properties were obtained from cancer surgery as documented in [12]. The three locations namely L1, L2 and L3 are labeled according to the distance between the tumor and the probe where L1 is the closest from the probe, L2 deeper than the first location L1 and L3 is the farthest to the probe. The magnitude and phase information were then recorded for the breast with the tumor. The data is then analysed with and without the tumor to decide whether or not a tumor is present. (a)

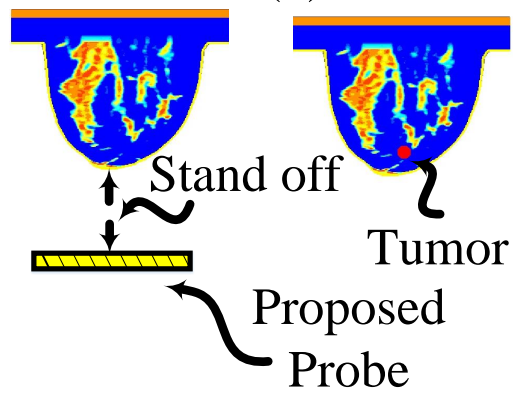

make the probe responses data sets of both testing with and without tumor easy to explore and visualize. Once the probe response of the two testing without and with tumor at the different locations are recorded ,the probe response was extracted and analyzed with and without PCA as shown in Fig. 4. The PCA feature extraction method is applied for both the healthy and the tumourous cases. The results of Fig. 4(a) show the magnitude of $S_{11}$ and Fig. 4(b) show the magnitude of $S_{11}$ using PCA. Fig. 4(c) shows the phase of $S_{11}$ and Fig. 4(d) shows the phase of $S_{11}$ using PCA. It is evident from the results that the difference in magnitude and phase of the reflection coefficient of the probe between the normal and tumourous case is greater for tumor locations that are closer to the probe. (a)

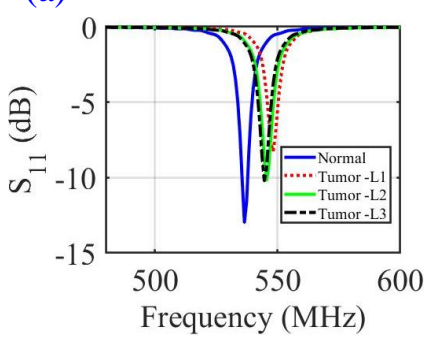

(c)

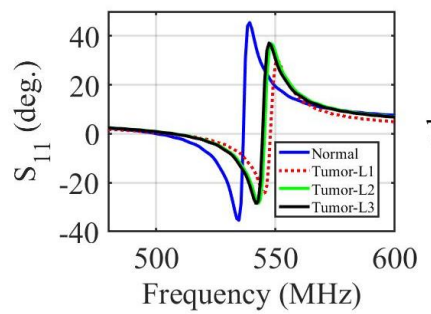

(b)

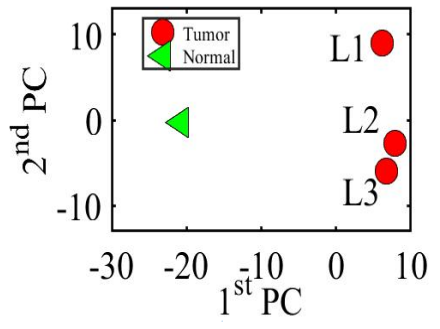

(d)

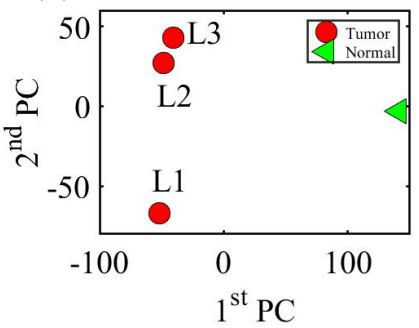

Fig. 4: Simulation results of the probe magnitude and phase response for normal breast tissue and breast tissue with $9 \mathrm{~mm}$ tumor at three different locations where: a) the magnitude of $S_{11}$, b) the magnitude of $S_{11}$ using PCA, c) the phase of $S_{11}$ and d) the phase of $S_{11}$ using PCA.

Then the simulation was extended to test the ability of the probe to detect various sizes of tumors in a single location. A tumor is inserted inside the same breast phantom with three different diameter sizes namely: $9 \mathrm{~mm}, 13 \mathrm{~mm}$, and $17 \mathrm{~mm}$, as shown in Fig. 3(b). The results are shown in Fig. 5 for tumor sizes of $9 \mathrm{~mm}, 13 \mathrm{~mm}$ and $17 \mathrm{~mm}$, respectively. In all the tumor sizes the probe was capable of detecting the presence of tumor tissues. Obviously from the results, larger tumor sizes are easier to detect.

\section{Conclusion}

In this work, We presented an alternative microwave modality for breast tumor detection using a single probe. The sensing mechanism is simple and has the advantage of being portable and comfortable for the user. Our numerical results have demonstrated the ability of sensing a tumor that is as small as $9 \mathrm{~mm}$ buried inside a normal realistic female breast phantom.

Fig. 3: Simulation setup: (a)Narrow band probe at distance off 5 $\mathrm{mm}$ from 3D normal realistic numerical breast phantom model in CST. (b)The embedded tumor in the normal numerical breast phantom.

Next, we employ the Principle Component Analysis (PCA) method to reduce the dimensionality of the problem by implementing a vector space transform [13]. PCA is feature extraction method [14] which used to emphasize variation of the probe responses data of both normal and tumours cases. The objective of PCA is to extract critical information from the frequency response data set and to express this information as a set of orthogonal variables called principal components [15]. Thus, via mathematical projection, high dimensional original data sets can be reduced to small number of variables without losing much of the original information to analyze trends, patterns and outliers [16, 14]. Moreover, PCA is used to

\section{Acknowledgments}

The authors acknowledge the financial support of Hadhramout Establishment for Human Development, Hadhramout, Yemen and Engineering Research Council (NSERC) of Canada.

\section{References}

[1] A. C. Society. (2018) Cancer facts and figures 2018 @ONLINE. [Online]. Available: https://www.cancer.org/content/dam/cancer- 
(a)

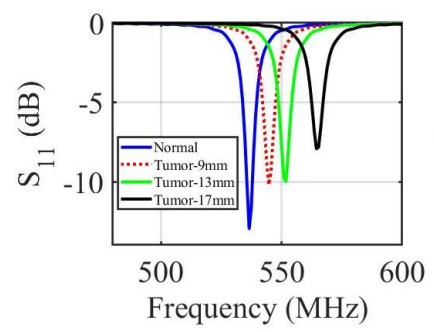

(c)

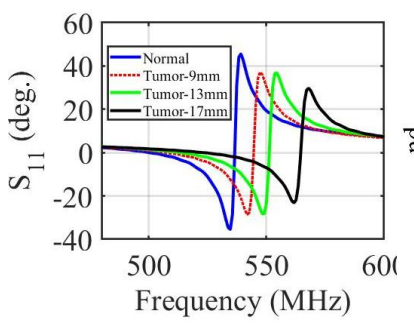

(d)

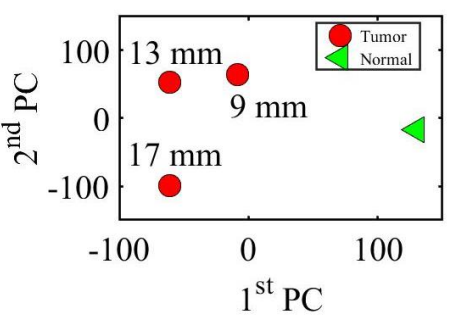

Fig. 5: Simulation results of using three different sizes of breast tumors of size $9 \mathrm{~mm}, 13 \mathrm{~mm}$ and $17 \mathrm{~mm}$ embedded in healthy breast phantom. where: a) the magnitude of $S_{11}$, b) the magnitude of $S_{11}$ using PCA, c) the phase of $S_{11}$ and d) the phase of $S_{11}$ using PCA.

org/research/cancer-facts-and-statistics/annual-cancerfacts-and-figures/2018/cancer-facts-and-figures-2018.pdf

[2] L. Wang, "Early diagnosis of breast cancer," Sensors, vol. 17, no. 7, p. 1572, 2017.

[3] M. Lazebnik, L. McCartney, D. Popovic, C. B. Watkins, M. J. Lindstrom, J. Harter, S. Sewall, A. Magliocco, J. H. Booske, M. Okoniewski et al., "A large-scale study of the ultrawideband microwave dielectric properties of normal breast tissue obtained from reduction surgeries," Physics in medicine and biology, vol. 52, no. 10, p. 2637, 2007.

[4] T. Sugitani, S.-i. Kubota, S.-i. Kuroki, K. Sogo, K. Arihiro, M. Okada, T. Kadoya, M. Hide, M. Oda, and T. Kikkawa, "Complex permittivities of breast tumor tissues obtained from cancer surgeries," Applied Physics Letters, vol. 104, no. 25, p. 253702, 2014.

[5] A. Martellosio, M. Pasian, M. Bozzi, L. Perregrini, A. Mazzanti, F. Svelto, P. E. Summers, G. Renne, L. Preda, and M. Bellomi, "Dielectric properties characterization from 0.5 to $50 \mathrm{ghz}$ of breast cancer tissues," IEEE Transactions on Microwave Theory and Techniques, vol. 65, no. 3, pp. 998-1011, 2017.

[6] V. Gazhonova, 3D Automated Breast Volume Sonography: A Practical Guide. Springer, 2016.

[7] J.-H. Chen, S. Chan, D.-C. Yeh, P. T. Fwu, M. Lin, and M.Y. Su, "Response of bilateral breasts to the endogenous hormonal fluctuation in a menstrual cycle evaluated using $3 \mathrm{~d}$ mri," Magnetic resonance imaging, vol. 31, no. 4, pp. 538-544, 2013.

[8] CST. (2017, Sep.) Computer simulation technology. cst computer simulation technology ag@ONLINE. [Online]. Available: http://www.CST.com

[9] UWCEM. (2017, Aug.) Breast phantom repository@ONLINE. [Online]. Available: http://uwcem.ece.wisc.edu/phantomRepository.html

[10] E. Zastrow, S. Davis, M. Lazebnik, F. Kelcz, B. Van Veem, and S. Hagness, "Database of $3 d$ grid-based numerical breast phantoms for use in computational electromagnetics simulations," Department of Electrical and Computer Engineering University of Wisconsin-Madison, 2008.
[11] E. Zastrow, S. K. Davis, M. Lazebnik, F. Kelcz, B. D. Van Veen, and S. C. Hagness, "Development of anatomically realistic numerical breast phantoms with accurate dielectric properties for modeling microwave interactions with the human breast," IEEE Transactions on Biomedical Engineering, vol. 55, no. 12, pp. 2792-2800, 2008.

[12] M. Lazebnik, D. Popovic, L. McCartney, C. B. Watkins, M. J. Lindstrom, J. Harter, S. Sewall, T. Ogilvie, A. Magliocco, T. M. Breslin et al., "A large-scale study of the ultrawideband microwave dielectric properties of normal, benign and malignant breast tissues obtained from cancer surgeries," Physics in medicine and biology, vol. 52, no. 20, p. 6093, 2007.

[13] M. Richardson, "Principal component analysis," URL: http://people. maths. ox. ac. uk/richardsonm/SignalProcPCA. pdf (last access: 3.5. 2013). Aleš Hladnik Dr., Ass. Prof., Chair of Information and Graphic Arts Technology, Faculty of Natural Sciences and Engineering, University of Ljubljana, Slovenia ales.hladnik@ntf.uni-lj.si, 2009.

[14] I. T. Jolliffe and J. Cadima, "Principal component analysis: a review and recent developments," Phil. Trans. R. Soc. A, vol. 374, no. 2065, p. 20150202, 2016.

[15] H. Abdi and L. J. Williams, "Principal component analysis," Wiley interdisciplinary reviews: computational statistics, vol. 2, no. 4, pp. 433-459, 2010.

[16] M. Richardson, "Principal component analysis," URL: http://people. maths. ox. ac. uk/richardsonm/SignalProcPCA. pdf (last access: 3.5. 2013). Aleš Hladnik Dr., Ass. Prof., Chair of Information and Graphic Arts Technology, Faculty of Natural Sciences and Engineering, University of Ljubljana, Slovenia ales.hladnik@ntf.uni-lj.si, 2009. 